\title{
Optical spectroscopy of the 2005 outburst of the X-ray transient XTE J1118+480
}

\author{
P. Elebert ${ }^{1}$, P. J. Callanan ${ }^{1}$ and M. A. P. Torres ${ }^{2}$ \\ ${ }^{1}$ Department of Physics, University College Cork, Ireland \\ email: p.elebert@ucc.ie \\ ${ }^{2}$ Harvard-Smithsonian Center for Astrophysics, \\ 60, Garden St., Cambridge, MA 02138, USA.
}

\begin{abstract}
We present time-resolved spectroscopy from the January 2005 outburst of the X-ray nova XTE J1118+480. X-ray observations show this outburst to be about half as bright as the previous outburst in 2000. This suggests that the accretion rate was lower than that in the 2000 outburst. Our spectroscopic analysis shows that the emission from the $\mathrm{H} \alpha$ line occurs at a higher velocity in the recent outburst compared with the 2000 outburst, more consistent with quiescence/near-quiescence values. This is surprising, considering the $\sim 5$ magnitude brightening of the accretion disk relative to its quiescent state.
\end{abstract}

Keywords. X-rays: binaries, accretion disks, techniques: spectroscopic

\section{Introduction}

The X-ray nova (XRN) XTE J1118+480, a black hole candidate, was discovered in March 2000 by the All-Sky Monitor on the Rossi X-ray Timing Explorer (Remillard et al. 2000). The optical counterpart was found to have brightened by $\sim 6$ magnitudes compared with the pre-outburst luminosity. Further analysis showed that the system underwent a previous mini-outburst in January 2000.

The primary has been determined to have a minimum mass of $6 \mathrm{M}_{\odot}$ (McClintock et al. 2001) which is greater than the theoretical maximum mass for a neutron star (Rhoades and Ruffini 1974). An upper limit of $0.36 \mathrm{M}_{\odot}$ has been estimated for the mass of the secondary, a K5 dwarf (McClintock et al. 2003).

The system remained in quiescence until January 2005, when it again increased in brightness both optically and in X-rays. This recent outburst closely resembles the minioutburst of January 2000 in terms of the length of the outburst. However, the maximum X-ray flux from the 2005 outburst is approximately half as strong as that from the 2000 outburst. Due to the rarity of their outbursts, there are relatively few detailed studies of XRN accretion disks during outburst.

\section{Data}

Our primary data consist of optical spectra acquired with the FAST spectrograph (Fabricant et al. 1998), attached to the $1.5 \mathrm{~m}$ Tillinghast Telescope at the Fred L. Whipple Observatory, Arizona. These data were acquired as part of the CfA ToO program on Xray Novae/Transients. Use of a 3 arcsec slit and the 300 line $\mathrm{mm}^{-1}$ grating provided a wavelength coverage of $\lambda \lambda 3480-7400 \AA$ with a dispersion of $\sim 1.46 \AA \mathrm{pix}^{-1}$. The spectra were extracted and wavelength calibrated using standard IRAF tasks as part of the CfA spectroscopic data pipeline. These data were obtained over 3 nights in January and February 2005. 


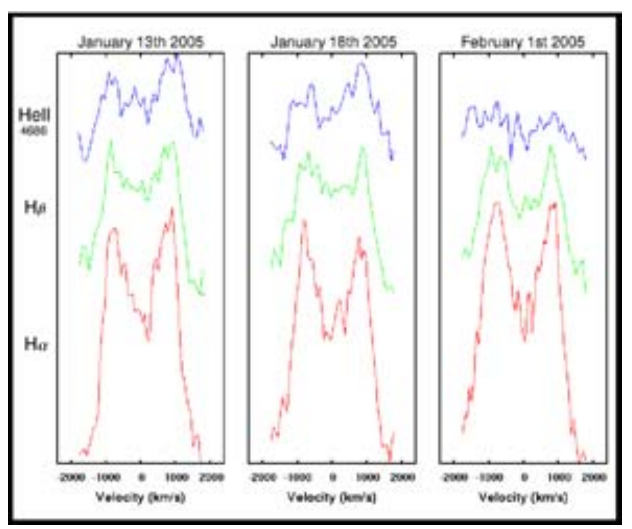

Figure 1. Averaged spectra for $\mathrm{H} \alpha, \mathrm{H} \beta$ and HeII $\lambda 4686$

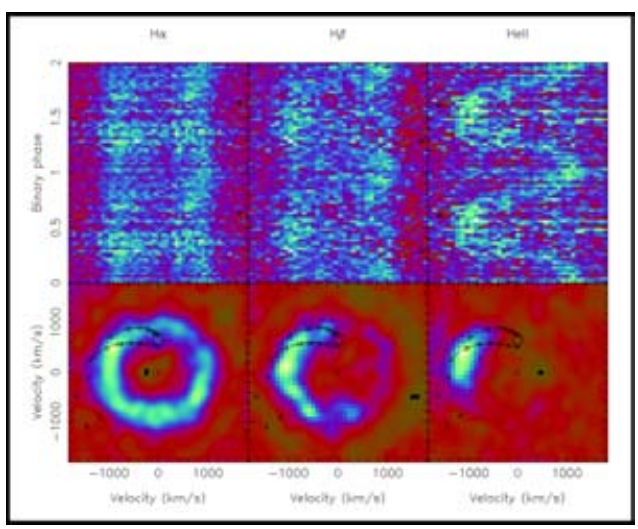

Figure 2. Trailed spectra and Doppler maps for Jan 132005

\section{Results and Discussion}

In Figure 1, the equivalent width of the HeII line is lower on the third night than on the first two nights. This may be consistent with the reduced X-ray flux (that powers this line) on the third night. 2-Gaussian fits to the averaged $\mathrm{H} \alpha$ lines gives a peak-to-peak separation of $\sim 1600 \mathrm{~km} \mathrm{~s}^{-1}$, also evident in the emission rings in the Doppler maps in Figure 2 (Marsh and Horne 1988).

In the 2000 outburst, the peak-to-peak separation of the $\mathrm{H} \alpha$ line was $\sim 1200 \mathrm{~km} \mathrm{~s}^{-1}$, and remained close to this value during that outburst (Torres et al. 2002). In quiescence however, the peak-to-peak velocity increased to $\sim 1660-1830 \mathrm{~km} \mathrm{~s}^{-1}$ (Torres et al. 2004). The higher peak-to-peak separation suggests that the outer part of the disk is less luminous during the recent outburst. reducing the amount of lower velocity material contributing to the line profiles, consistent with our observations. This suggests a considerably lower accretion rate in the January 2005 outburst, which is surprising, considering the $\sim 5$ magnitude optical brightening of the accretion disk relative to its quiescent state.

\section{Acknowledgements}

The ToO program at CfA also involved the following CoIs: M. R. Garcia, D. Steeghs, J. E. McClintock, J. M. Miller and P. Zhao. We acknowledge the use of molly, doppler and trailer software packages developed by T. R. Marsh, University of Warwick.

P. E. and P. J. C. acknowledge support from Science Foundation Ireland.

\section{References}

Fabricant, D., Cheimets, P., Caldwell, N. \& Geary, J. 1998 PASP 110, 79

Marsh, T. R. \& Horne, K. 1988 MNRAS 236, 269

McClintock, J. E., Garcia, M. R., Caldwell, N., Falco, E. E., Garnavich, P. M. \& Zhao, P. 2001 ApJ 551, 147

McClintock, J. E., Narayan, R., Garcia, M. R., Orosz, J. A., Remillard, R. A. \& Murray, S. S. 2003 ApJ 593, 435

Remillard, R., Morgan, E., Smith, D. \& Smith, E. 2000 IAU Circ. 7389

Rhoades, C. E. \& Ruffini, R. 1974, Phys. Rev. Lett. 32, 324

Torres, M. A. P., Callanan, P. J., Garcia, M. R., McClintock, J. E., Garnavich, P., Balog, Z., Berlind, P., Brown, W. R., Calkins, M. \& Mahdavi, A. 2002 ApJ 569, 423

Torres, M. A. P., Callanan, P. J., Garcia, M. R., Zhao, P., Laycock, S. \& Hong, A. K. H. 2004 ApJ 612, 1026 